\title{
Suitable reference genes for relative quantification of miRNA expression in prostate cancer
}

\author{
Annika Schaefer ${ }^{1,2,3}$, Monika Jung ${ }^{1}$, \\ Kurt Miller ${ }^{1}$, Michael Lein ${ }^{1,2}$, Glen Kristiansen ${ }^{4}$, \\ Andreas Erbersdobler ${ }^{5}$ and Klaus Jung ${ }^{1,2,6}$

\section{${ }^{1}$ Department of Urology} \\ University Hospital Charité \\ ${ }^{2}$ Berlin Institute for Urologic Research \\ ${ }^{3}$ Department of Biology \\ Chemistry and Pharmacy \\ Free University Berlin \\ Berlin, Germany \\ ${ }^{4}$ Department of Surgical Pathology \\ University Hospital Zurich \\ Zurich, Switzerland \\ ${ }^{5}$ Department of Pathology \\ University Hospital \\ Rostock, Germany \\ ${ }^{6}$ Corresponding author: Tel, 4930-450-515041; \\ Fax, 4930-450-515904; E-mail, klaus.jung @ charite.de \\ DOI 10.3858/emm.2010.42.11.076
}

Accepted 29 September 2010

Available Online 1 October 2010

Abbreviations: $\mathrm{Cq}$, quantification cycle; $\mathrm{CV}$, coefficient of variation; hsa-miR, microRNA from Homo sapiens; miRNA, microRNA; RT-qPCR, quantitative real-time reverse transcription polymerase chain reaction

\begin{abstract}
Real time quantitative PCR (qPCR) is the method of choice for miRNA expression studies. For relative quantification of miRNAs, normalization to proper reference genes is mandatory. Currently, no validated reference genes for miRNA qPCR in prostate cancer are available. In this study, the expression of four putative reference genes (hsa-miR-16, hsa-miR-130b, RNU6-2, SNORD7) was examined with regard to their use as normalizer. After SNORD7 was already shown an inappropriate reference gene in preliminary experiments using total RNA pools, we studied the expression of the putative reference genes in tissue and normal adjacent tissue sample pairs from 76 men with untreated prostate carcinoma collected after radical prostatectomy. hsa-miR-130b and RNU6-2 showed no significantly different expression between the matched malignant and
\end{abstract}

non-malignant tissue samples, whereas hsa-miR-16 was significantly underexpressed in malignant tissue. Softwares geNorm and Normfinder predicted hsamiR-130b and the geometric mean of hsa-miR-130b and RNU6-2 as the most stable reference genes. Normalization of the four miRNAs hsa-miR-96, hsamiR-125b, hsa-miR-205, and hsa-miR-375, which were previously shown to be regulated, shows that normalization to hsa-mir-16 can lead to biased results. We recommend using hsa-miR-130b or the geometric mean of hsa-miR-130b and small RNA RNU6-2 for normalization in miRNA expression studies of prostate cancer.

Keywords: gene expression profiling; microRNA; polymerase chain reaction; prostate neoplasms

\section{Introduction}

MicroRNAs (miRNAs) are small non-coding RNAs with a length of approximately 22 nucleotides. They regulate gene expression by mRNA cleavage and at posttranscriptional level by translational suppression and play important roles in various biological and metabolic processes (Min and Yoon, 2010). Recent studies have demonstrated their decisive significance in tumor development and metastasis (Garzon et al., 2009). Bioinformatic predictions indicate that miRNAs regulate $\sim 30 \%$ of all protein coding genes (Filipowicz et al., 2005). It is estimated that approximately 1,000 miRNAs exist in the vertebrate genome (Berezikov et al., 2005). So far, 940 human miRNAs are registered at miRBase release 15.0 (http://microrna.sanger.ac. uk/; access: July 6, 2010).

The differential expression of miRNAs depends on tissue type and disease status suggesting their potential not only as new diagnostic, prognostic, and predictive markers, but also as targets of novel therapeutic strategies (Lee and Dutta, 2009). Therefore, there is a growing interest in miRNA profiling in order to identify specific miRNAs with diagnostic/therapeutic value, and a wealth of miRNA studies can be expected for the near future, which will be performed similarly as we have seen in the numerous mRNA expression studies of the last decade. The methods used to study miRNA expression are Northern Blotting, 
bead-based flow cytometry, microarray technology, and quantitative real-time reverse transcriptionpolymerase chain reaction (RT-qPCR) (Bartels and Tsongalis, 2009). This latter technique is the method of choice for analysis of mRNA expression in biological material (Nolan et al., 2006) and new techniques have also offered the possibility of measuring expression of miRNAs by this technique in recent years (Chen et al., 2005). QPCR combines the advantages of high sensitivity and specificity and can be used in high-throughput approaches. To study changes in a gene of interest the relative quantification of expression is the generally accepted approach in comparison with the absolute quantification working with absolute concentrations based on an external or internal calibration curve (Bustin et al., 2009). In relative quantification, the expression of a target gene is normalized to the expression of one or more stably expressed endogenous reference genes simultaneously measured in the same sample.

However, several criteria have to be fulfilled to render a gene eligible as a reference gene (Vandesompele et al., 2002; Bustin and Nolan, 2004). It should be expressed at a constant non-regulated level independent of biological variations, disease stages or treatments. The stability of a given reference gene has to be experimentally validated for each study design. The range of expression should be similar to that of the target gene in order to be able to measure both genes in one sample dilution. The reference gene should be of similar length as the gene of interest to guarantee the same efficiencies during RNA isolation and reverse transcription.

In miRNA research, suitable reference genes, especially for expression studies in prostate cancer, have not been satisfyingly identified. Only a few studies have investigated the question of reference gene selection in miRNA RT-qPCR. Small nucleolar RNAs such as RNU6-2 (RNA, U6 small nuclear 2; alias RNU6B), SNORD7 (small nucleolar RNA C/D box 7; alias Z30), and SNORD43 (small nucleoalr RNA C/D box 43; alias RNU43) are generally used for normalization of miRNA expression studies (Ozen et al., 2008; Leite et al., 2009; Spahn et al., 2009; Chang et al., 2010). Some miRNAs have also been described as reference genes for a set of tissues from solid tumors (Peltier and Latham, 2008) as well as for breast cancer (Davoren et al., 2008). Recently, it has been proposed that normalizing to the mean expression of all tested miRNAs is superior to normalization to one or a pair of stably expressed small RNAs (Mestdagh et al., 2009). Yet, this approach is limited, as it is only feasible if a large number of miRNAs is studied, which is not always possible, especially in functional studies.

We performed a PubMed literature search to identify suitable reference genes for miRNA qPCR of prostate cancer specimen. We identified 16 articles (Jiang et al., 2005; Mattie et al., 2006; Porkka et al., 2007; Ambs et al., 2008; Josson et al., 2008; Mitchell et al., 2008; Ozen et al., 2008; Place et al., 2008; Prueitt et al., 2008; Rokhlin et al., 2008; Gandellini et al., 2009; Leite et al., 2009; Noonan et al., 2009; Siva et al., 2009; Spahn et al., 2009; Tong et al., 2009), in which miRNA RT-qPCR was performed. In 14 articles, relative quantification was performed and the reference genes utilized were specified. The most commonly used reference gene was the small RNA RNU6-2 which was used in 4 studies (Jiang et al., 2005; Ambs et al., 2008; Prueitt et al., 2008; Noonan et al., 2009). Further reference genes or combination of reference genes were: GAPDH (Place et al., 2008; Rokhlin et al., 2008) and RNU6-2/SNORD43 (Ozen et al., 2008; Spahn et al., 2009) (each twice), SNORD43 (Leite et al., 2009), TATA box binding protein mRNA (Porkka et al., 2007), RNU62/miR-16 (Josson et al., 2008), RNU6-2/RNaseP (Gandellini et al., 2009), and let-7/miR-16 (Mattie et al., 2006) (each once). The reasons for selection of the reference genes were only given in three studies, but these were not satisfying (Jiang et al., 2005; Mattie et al., 2006; Josson et al., 2008). This search clearly showed that an unequivocal recommendation for miRNA reference genes for prostate cancer is lacking and a study concerning this issue has not been performed until now. On the other hand, there has been a lack of agreement regarding differentially expressed miRNAs in prostate cancer(Schaefer et al., 2010b). The use of unvalidated reference genes seems to be one of the major reasons for the discrepancies in miRNA expression results between the previously published studies (Schaefer et al., 2010b).

Using the main selection criteria of good measurability and stable expression in non-malignant and malignant tissue independent of the stage or grade of the tumor, we decided to investigate four putative reference genes, two miRNAs (hsa-miR16 and hsa-miR-130b) and two small RNAs (RNU6-2 and SNORD7). As mentioned above, hsa-miR-16 and RNU6-2 were frequently, but not always well-founded in their use as reference genes in prostate cancer tissue or prostate cancer cell lines (Jiang et al., 2005; Mattie et al., 2006; Ambs et al., 2008; Josson et al., 2008; Noonan et al., 2009; Ozen et al., 2008; Prueitt et al., 2008; Gandellini et al., 2009; Spahn et al., 2009), SNORD7 is commercially distributed as reference 


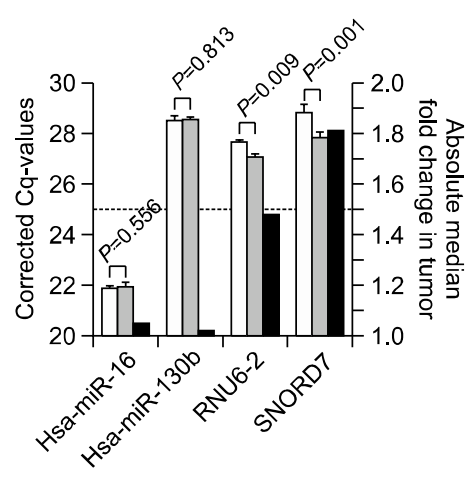

Figure 1. Expression of reference gene candidates measured in RNA pools obtained from matched non-malignant and malignant prostate cancer tissue specimens. For each pool, RNA extracts were diluted to the same concentration and then equal volumes were mixed to obtain the same final concentration in the RNA pools $(250 \mathrm{ng} / \mu \mathrm{l})$ from malignant and non-malignant samples, respectively. The first two columns of every gene represent the expression values as mean Cq-values $\pm 1 \mathrm{SD}(n=$ 3; blank, non-malignant tissue; grey, malignant tissue) related to the left $y$-axis scale. The third column (black) represents the median fold change between pools from non-malignant and malignant tissue related to the right $y$-axis scale. The arbitrary line at 1.5 represents the rough estimate of differential expression between non-malignant and malignant tissue, as explained in the text.

gene and recommended as generally stable reference gene (Pradervand et al., 2009), and we recently suggested hsa-miR-130b for normalization (Schaefer et al., 2010a). Since it has been proposed that normalization should be done on a set of reference genes (Vandesompele et al., 2002), we also tested the geometric mean of hsa-miR$130 \mathrm{~b}$ and RNU6-2 as an approach for miRNA normalization.

\section{Results}

\section{Expression of candidate reference genes}

To evaluate the measurability of the putative reference genes hsa-miR-16, hsa-miR-130b, RNU6-2, and SNORD7 and to obtain a rough estimate of their expression stability in malignant and nonmalignant tissue, the expression levels of these genes were determined in RNA pools prepared from normal and malignant tissue samples. For each pool, the same volumes of 12 samples of equal RNA concentration were combined as described in the Methods section. All reference gene candidates were expressed at suitable levels, showing good measurability (Figure 1). Hsa-miR16 had the highest expression with mean Cqvalues below 25. Expression of SNORD7 was the lowest, but still with Cq-values below 30 , showing median fold changes of 1.81 between pools (Figure 1 ; 3rd column). We decided to use a
Table 1. Correlation of the expression of putative reference genes with tumor stage and Gleason score.

\begin{tabular}{lcccccc}
\hline \multirow{2}{*}{$\begin{array}{l}\text { Reference } \\
\text { gene approach }\end{array}$} & \multicolumn{2}{c}{$\mathrm{pT}$ stage } & & \multicolumn{2}{c}{ Gleason score } \\
\cline { 2 - 3 } \cline { 6 - 6 } & $r^{\mathrm{a}}$ & $P$ value & & \multicolumn{1}{c}{$r$} & $P$ value \\
\hline Hsa-miR-16 & -0.007 & 0.95 & & -0.184 & 0.11 \\
Hsa-miR-130b & -0.049 & 0.67 & & -0.151 & 0.19 \\
RNU6-2 & -0.050 & 0.67 & & 0.070 & 0.51 \\
Geometric mean of & -0.067 & 0.57 & & -0.022 & 0.86 \\
Hsa-miR-130b/RNU6-2 & & & & \\
\hline${ }^{\text {a }}$ Pearson correlation coefficient. & & & & \\
\end{tabular}

change limit of 1.5 as an indicator of differential expression. The high arbitrary threshold was chosen to minimize risk of type II errors. According to that criterion SNORD7 was excluded from further analyses. However, RNU6-2 showed a mean fold change of 1.48 but was included in the subsequent, more detailed search for suitable reference genes despite its differential expression (Figure 1).

The expression of the remaining candidate genes (hsa-miR-16, hsa-miR-130b, and RNU6-2) was then determined in 76 matched pairs of malignant and non-malignant tissue specimens obtained from non-treated prostate carcinomas after radical prostatectomy. For statistical analyses, Cq-values were normalized to qPCR efficiency and interplate controls using the software GenEx (Kubista et al., 2006). Since the expression of candidate reference genes did not correlate with the Gleason score or tumor stage as the two main factors that may determine the expression behavior (Table 1), all values were merged, independent of the stage and grade of the tumor, into one data set for further calculations. There were no significant differences between Cq-values in the tissue groups for hsa-miR-130b and RNU6-2, whereas hsa-miR-16 was significantly underexpressed in malignant tissues (Figure 2; $P=0.0002$, paired $t$-test). Thus, hsa-miR-16 was excluded on this account from further analyses since the high number of samples investigated and the high significance level allowed both the risk of type I and II errors to be excluded with relatively high certainty.

The fact that a reference gene shows no statistical difference between groups does not mean that its expression is equal in those groups. Therefore, we also tested the equivalence of the expression values of hsa-miR-130b, RNU6-2 and the geometric mean between the malignant and non-malignant sample group (Haller et al., 2004). The approved difference between the groups was 


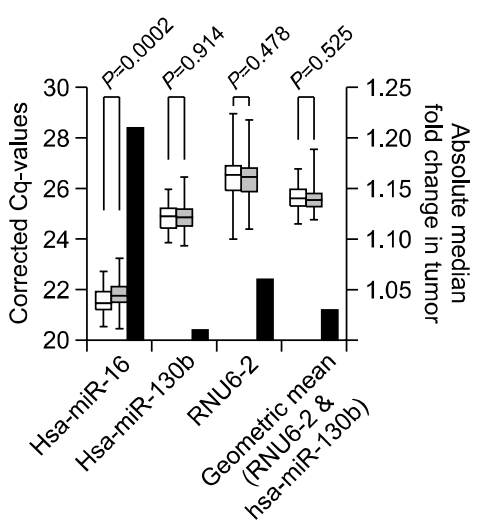

Figure 2. Expression of reference gene candidates in matched non-malignant and malignant sample pairs from 76 prostate cancer tissue specimens. Left y-axis: Expression values are given as Cq-values, which were corrected to efficiency and two interplate controls. Boxes (blank:, non-malignant tissue; grey: malignant tissue) represent lower and upper quartiles with the median as a horizontal line and the mean as a cross. The whiskers represent the ranges of expression. Only hsa-miR-16 expression was significantly different between normal and malignant tissue $(P=0.0002$, $t$-test of paired data). Right y-axis: The columns (black) represent the median fold changes between the malignant and non-malignant tissue samples.

set to 0.26 for all three reference genes, which equates to a fold change of 1.2. The expression of RNU6-2 in both tissue groups was not equal, when using this cut-off, whereas both hsa-miR-130b and the geometric mean showed equivalent values in the malignant and non-malignant samples.

\section{Stability of candidate reference genes}

The overall stability of the expression is a further major criterion for a good reference gene. The variance of a reference gene over all groups is a measure of this stability. We compared the variances of hsa-miR-130b and RNU6-2 expression and the geometric mean of both candidates. RNU6-2 had a significantly higher variance than hsa-miR-130b or the geometric mean $(P<0.0001$, Levene's test), whereas the variances of hsamiR-130b and the geometric mean did not differ significantly $(P=0.52$, Levene's test).

The stability of candidate reference genes was further assessed by the Normfinder and geNorm software (Vandesompele et al., 2002; Andersen et al., 2004). GeNorm calculates the stability values (M-values) for all candidate genes and excludes the candidate with the lowest stability and recalculates the stability value, until the two most stable genes are predicted. In our study, M-values below 1.5 were defined in the program as the critical limit. The geometric mean and hsa-miR-130b were the two most stable genes with an M-value of 0.47 (Figure 3A). Normfinder also calculates a stability value, named the variability, for each gene. It estimates the single most stable gene and the best combination of two genes. In contrast to geNorm it can account for group differences. Normfinder analysis identified hsa-miR-130b as the single most stable gene with a variability of 0.078 . The geometric mean of hsa-miR-130b and RNU6-2 lowered the variability to 0.027 , substantiating the increased stability (Figure 3B).

\section{Effect of normalization on relative quantification of miRNA expression of genes of interest}

The effect of normalization using either of these three reference gene candidates or hsa-miR-16, which is itself regulated in prostate cancer, on the

\section{A}

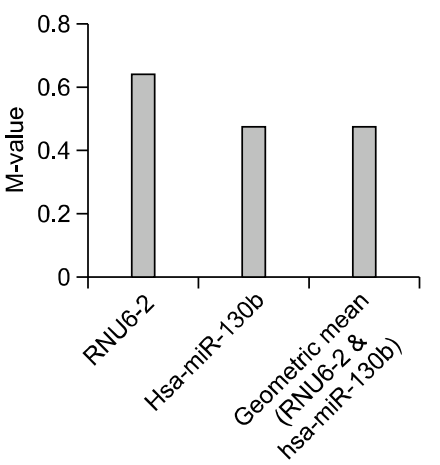

B

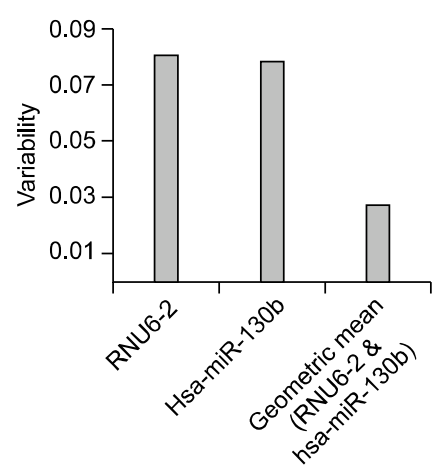

Figure 3. Stability analyses of selected candidate reference genes in the geNorm and Normfinder programs. (A) Stability value M of candidate reference genes calculated by geNorm. (B) Variability of candidate reference genes calculated by Normfinder. Highly stable expression of genes is indicated by a low $\mathrm{M}$ value in geNorm and a low variability value in Normfinder, respectively. 


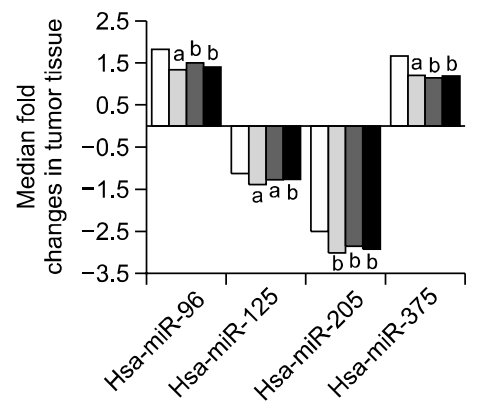

Figure 4. Quantitative differences in miRNA expression in prostate cancer by normalization with different reference gene approaches. The median fold changes of hsa-miR-96, hsa-miR-125b, hsa-miR-205, and hsa-miR-375 expression in 76 malignant tissue samples in comparison to matched normal tissue are presented. Expression was either normalized to hsa-miR-16 (blank columns), RNU6-2 (light grey columns), hsa-miR-130b (medium grey columns) or the geometric mean of hsa-miR-130b and RNU6-2 (black columns). There were no significant differences in any fold changes of expression for the four target miRNAs when they were normalized against either RNU6-2, hsa-miR- $130 \mathrm{~b}$ or the geometric mean of hsa-miR-130b and RNU6-2 (Friedman' test with Dunn's post test, $P=0.810$ ). In contrast, the fold changes of all four miRNAs normalized against hsa-miR-16 were significantly different from the fold changes obtained with the normalization against has-miR- $130 \mathrm{~b}$, RNU6-2 or the geometric mean of hsa-miR-130b and RNU6-2. Significance levels are indicated by letters at the corresponding columns: ${ }^{a}, P<0.005 ;{ }^{b}, P<0.0005$ (Wilcoxon test of matched paired samples).

expression results for hsa-miR-96, hsa-miR-125b, hsa-miR-205, and hsa-miR-375 was tested. These four miRNAs of interest were previously shown to be differentially regulated in prostate cancer (Schaefer et al., 2010a). Comparison of the four normalization strategies demonstrated that fold changes are indeed significantly influenced by the normalization approach (Figure 4). Fold changes after normalization to hsa-miR-130b, RNU6-2 or their geometric mean did not differ from each other (Friedman' test with Dunn's post test, $P=0.810$ ), but the fold changes of all four miRNAs of interest normalized with hsa-miR-16 were significantly different from those related to the other three normalization approaches (Figure 4; Wilcoxon test, always $P$ value $<0.01)$.

As mentioned above, the variation in expression of a given gene is composed of biological and technical variation and it is the technical variation that should be reduced by normalizing with the reference genes. Therefore, normalization to a more efficient reference gene should result in lower coefficients of variation (CV) in the expression data of the miRNA of interest. The CVs of the miRNAs normalized with RNU6-2 were significantly higher than those for the same miRNAs normalized with hsa-miR-130b or the geometric mean of hsa-miR$130 \mathrm{~b}$ and RNUB6 (Figure 5; $P=0.0009$, Friedman test with Dunn's post test).

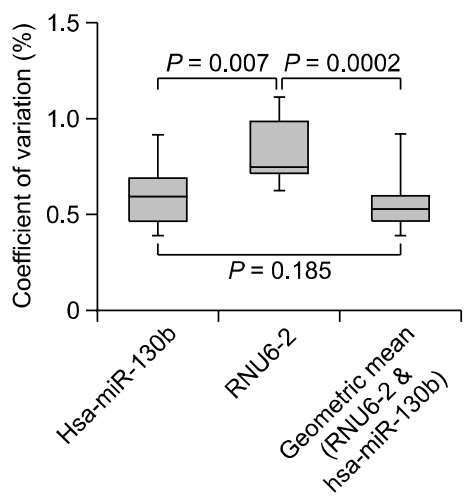

Figure 5. Effect of the normalization approach on the coefficient of variation of expression for four miRNAs of interest in prostate cancer. Hsa-miR-96, hsa-miR-125b, hsa-miR-205, and hsa-miR-375 were measured in 76 malignant and matched normal tissues samples. Each group was either normalized to hsa-miR-130b, RNU6-2 or the geometric mean of both. The coefficient of variation (CV) was calculated for each miRNA and tissue group. The box represents the lower and upper quartiles of the $\mathrm{CV}$ with the median as a horizontal line and the mean as a cross for normalization to one of the three reference genes. The whiskers represent the range of CVs. Significances were calculated by $t$-test of paired data.

\section{Discussion}

The relative quantification of gene expression by qPCR has become one of the major methods for studying miRNA expression in tissues or cell cultures, but the worth of this in expression studies is strongly dependent on the selection of stably expressed reference genes. In this study, we present the first experimentally validated comparison of reference genes for normalization of miRNA expression in prostate cancer. To date, the question of which reference genes should be used for the relative quantification of miRNA expression studies has not been systematically investigated. Nevertheless, it is mandatory to validate the expression of reference genes for each study design, as normalization to an unsuitable reference gene has been shown to lead to biased results (Dheda et al., 2005; Ohl et al., 2005). In the majority of miRNA expression studies, miRNAs or other small RNAs were used as reference genes, but some studies also normalized expression to longer mRNAs, namely GAPDH, TATA box binding protein mRNA, and RNAseP (Porkka et al., 2007; Huang et al., 2008; Place et al., 2008; Rokhlin et al., 2008; Gandellini et al., 2009). It is questionable as to whether mRNAs can be used as reference genes in miRNA expression studies, because longer RNAs may have different isolation efficiencies compared to miRNAs (Peltier and Latham, 2008), they may be degraded faster due to their length, and they are reverse transcribed with different 
techniques than the miRNAs of interest. GAPDH has also been shown to be inferior to other reference genes (Ohl et al., 2005) and even to be regulated in late pathological stages of prostate cancer (Rondinelli et al., 1997).

We investigated the expression of RNU6-2, hsa-miR-16, hsa-miR-130b in 76 matched normal and malignant prostate cancer tissues. We demonstrated that hsa-miR-130b is equally and stably expressed in normal and malignant prostate tissue and has a low expression variance across all samples.

Hsa-miR-16 has previously been used as a reference gene (Mattie et al., 2006; Josson et al., 2008) and has been reported to be similarly expressed in PC3 and LNCaP cells (Mattie et al., 2006). However, hsa-miR-16 expression is significantly regulated in prostate cancer tissue in comparison to normal tissue as shown in this study and also demonstrated by others (Porkka et al., 2007; Bonci et al., 2008). This regulation leads to erroneous results when other miRNAs are normalized to its expression. Due to its under-expression, overexpressed miRNAs seem to be much more highly expressed, whereas down-regulated miRNAs may not seem regulated at all (Figure 4). Therefore, the normalization of miRNA expression in prostate cancer tissue to hsa-miR-16 is more or less inappropriate.

RNU6-2 is the most common reference gene for miRNA expression studies. Two studies of miRNA expression in prostate cancer cell lines have reported RNU6-2 expression values across the studied cell lines and treatment groups (Jiang et al., 2005; Josson et al., 2008). Both studies showed that RNU6-2 is expressed at comparable levels but they did not provide statistical proof of their stable expression, they studied expression only in prostate cancer cell lines and not in tissue, and sample numbers were very low (four and five samples, respectively). Here we showed that RNU6-2 is not significantly regulated in prostate cancer. However, it did not prove to be equally expressed when a cut-off of a 1.2-fold allowed difference between tissue groups was used. The variance in RNU6-2 expression across both tissues was significantly higher than that of hsa-miR-130b. When hsa-miR-96, hsa-miR-125b, hsa-miR-205, and hsa-miR-375 that are some of the miRNAs of interest in prostate cancer research (Schaefer et al., 2010a) were normalized to RNU6-2 there were significantly higher CVs for normalized miRNAs in comparison to the CVs when their expression was normalized with hsa-miR-130b.

We also calculated the geometric mean of hsamiR-130b and RNU6-2 expression and used it for normalization according to Vandesompele et al. (Vandesompele et al., 2002). The geometric mean had a significantly lower variance than RNU6-2. Accordingly, the normalization of the expression of hsa-miR-96, hsa-miR-125b, hsa-miR-205, and hsa-miR-375 to the geometric mean also led to significantly lower CVs. Although variance and CV were significantly higher for RNU6-2 than for the geometric mean or hsa-miR-130b, we did not find that differences in the selected miRNAs between normal and malignant tissue were significantly altered when they were normalized to RNU6-2. Biased results could occur nevertheless, especially when only small samples are examined or when regulation of the miRNA of interest is low.

We also verified our results by analyzing the stability of candidate reference genes with geNorm or Normfinder. However, the results were limited. It is mandatory to use at least three non-regulated genes for analysis but five to ten are recommended (Andersen et al., 2004). As we only studied two non-regulated small RNAs in our panel we included the geometric mean of hsa-miR-130b and RNU6-2 in the analyses with geNorm and Normfinder, but this is not independent from the other genes and therefore may bias the results (Vandesompele et al., 2002). Nevertheless, the geNorm and Normfinder analyses supported our view that normalization to hsa-miR-130b and the geometric mean should be preferred to normalization to RNU6-2. Another limitation of this study was the small number of examined potential reference genes. Unfortunately, we were not able to investigate more reference genes in this sample set due to the limitation of available material. However, as the sample number in this study was comparably very high $(n=76)$, we can provide strong statistical reliability that the identified reference genes hsa-miR-130b and RNU6-2 are indeed stably expressed. This fact and the use of a relatively high arbitrary threshold of differential expression in the first round of our approach to select reference genes, as explained in the results, minimized the risk of type I and II errors of our results as far as possible. We believe that this result of a stably expressed reference gene pairs could be a useful starting point in searching for further reference genes.

The herein investigated reference genes are only recommended for miRNA RT-qPCR studies as all data are solely based on this platform. We checked for miR-130b and RNU6-2 expression in a publicly available dataset (GSE23022). While RNU6-2 was not available on the chip, miR-130b appeared to be slightly upregulated in the 20 prostate cancers and paired normal adjacent 
tissues. The discrepancies between both experiments can have several reasons. For example, differences in the detection platform, technical and experimental errors, and the use of different sample sets. In conclusion, the observed discrepancies underline that suitable reference genes for miRNA microarray need to be identified in an independent study.

Further, it is still under consideration, whether the use of reference genes is the most suitable normalization approach for miRNA microarray studies. Other methods are available, which do not rely on previously defined reference genes. Pradervand et al. (2009) developed a normalization approach based on the assumption that a set of miRNAs has the same expression across all arrays. These nonchanging miRNAs named invariants are identified for each experiment independently. Beside normalization to reference genes and invariants, other normalization strategies exist as quantile normalization or variance stabilizing normalization (Meyer et al., 2010), which are in part also used for mRNA arrays (Bolstad et al., 2003), but adapted to the specific needs for miRNA profiling. However, there is no general consent, which normalization is superior to the others (Pradervand et al., 2009).

In summary, the careful selection of reference genes plays a crucial role in gene expression studies. The candidate reference genes have to be validated for each study design, as it has been proven that even frequently used reference genes are not stable under different conditions. We would recommend using hsa-miR-130b as single reference gene or the geometric mean of hsa-miR-130b and RNU6-2 as a combined pair for relative quantification of miRNA expression studies in prostate cancer tissue.

\section{Methods}

\section{Patients and tissue samples}

Tumor tissue and adjacent normal tissue from 76 men with medically untreated prostate carcinoma were collected after radical prostatectomy (median age $63 \mathrm{yr}$, range 49-74 $\mathrm{yr}$; median pre-operative prostate-specific antigen concentration $6.7 \mu \mathrm{g} / \mathrm{l}$, range $0.92-41.9 \mu \mathrm{g} / \mathrm{l}$ ) and already used in a previous study (Schaefer et al., 2010a). Tumor stage was determined according to the International Union Against Cancer (Sobin and Wittekind, 2002). Forty-five tumors were staged as pT2 and 31 as pT3. Tumor grading was done according to Gleason (Epstein et al., 2005). Gleason score was distributed as follows: one times Gleason score 5, 31 times Gleason score 6, 30 times Gleason score 7, nine times Gleason score 8, and five times Gleason score 9 . Twenty-seven patients had positive surgical margins but none had metastases. The decisive selection of samples was based on the availability of cryo-preserved tissues.

The study was approved by the ethical board of the hospital. Fresh prostate tissues were sampled directly after surgical removal of the gland. One full frontal section, which was grossly tumor suspicious, was deep-frozen in liquid nitrogen. A diagnostic hematoxylin stain was prepared to verify tumor content and to identify areas of normal and tumor tissue. These regions of interest were punch biopsied, and then another frozen section was obtained to ascertain tumor content. Only cases with more than $90 \%$ tumor tissue were considered for further analysis (Schaefer et al., 2010a).

\section{RNA extraction}

Frozen matched malignant and non-malignant samples were collected in RNAlater Stabilization reagent (Qiagen $\mathrm{GmbH}$, Hilden, Germany). RNA was extracted with the miRNeasy Mini Kit (Qiagen) and the yield and A260/280 ratio were monitored with a NanoDropND-100 spectrometer (NanoDrop Technologies, Wilmington, DE). The mean A260/A280 ratio was $2.00 \pm 0.03$. RNA integrity numbers were measured with the 2100 Bioanalyzer using the RNA 6000 Nano Kit (Agilent Technologies, Santa Clara, CA). Only RNA extracts with an RNA integrity number $>6$ were included in further analysis. Mean values did not differ significantly $(P=0.30$, unpaired $t$-test $)$, with mean values of $7.15 \pm 0.62$ for normal tissue and $7.26 \pm$ 0.73 for malignant tissue. Further analytical details corresponded to specifications as reported previously (Jung et al., 2009; Schaefer et al., 2010a).

To assess the measurability of the expression levels of the reference genes, RNA pools were prepared from 12 RNA extracts obtained from matched paired malignant (pT2, $n=7 ; \mathrm{pT} 3, n=5$; Gleason score: two times 5 and 6 , 6 times 7, and two times 9) and non-malignant tissue specimens. For each pool, RNA extracts were diluted to the same concentration $(250 \mathrm{ng} / \mu \mathrm{l})$ and then equal volumes $(4.5 \mu \mathrm{l})$ were mixed to obtain the same final concentration in the RNA pools from malignant and non-malignant samples, respectively. The RNA concentrations of the original solutions and the dilutions as well as the final concentrations in the mixtures were monitored and verified by NanoDrop spectrophotometry so that both pools contained equivalent RNA amounts from every sample.

\section{MiRNA quantitative real-time RT-PCR}

For detection of mature miRNAs, a two-step qPCR was carried out using the TaqMan MiRNA Assay (Applied Biosystems, Foster City, CA) according to the manufacturer's protocol previously described. $6.67 \mathrm{ng}$ total RNA were reverse transcribed using miRNA-specific stemlooped primers, $10 \mathrm{nmol}$ dNTP mix, $2.6 \mathrm{U}$ RNase Inhibitor, 33.5 U MultiScribe RT enzyme, and $1 \times$ RT Buffer. RTqPCR measurements were performed on the Light Cycler 480 Instrument (Roche Diagnostics $\mathrm{GmbH}$, Mannheim, Germany; Software version 1.3.0) in white 96-well PCRplates with $10 \mu \mathrm{l}$ final volume including $1 \mu \mathrm{l}$ RNA-specific cDNA, $1 \times$ TaqMan Universal PCR Mastermix NoAmpErase UNG and gene-specific TaqMan MiRNA Real-Time PCR- 
Assay solution according to the manufacturer's protocol Samples were measured in triplicate and a no template control and two interplate controls were included in each PCR run. To minimize the analytical variation, paired malignant and non-malignant samples were always analyzed on one PCR plate. Analytical precision was assessed by intra-run measurements $(n=5)$ for each miRNA and ranged from 0.24 to $0.71 \%$ for quantification cycle $(\mathrm{Cq})$ values between 24.61 and 30.6.

GenEX software (MultiD Analyses AB, Göteborg, Sweden) was used to analyze and normalize the qPCR data (Kubista et al., 2006). Cq-values were corrected to PCR efficiencies $(E=0.825)$ and for differences between runs by normalizing with interplate calibrators.

$$
\begin{array}{r}
\text { Efficiency: } C_{E=100 \%}=C q_{E} \frac{\log (1+E)}{\log 2} \\
\text { Interplate normalization: } C_{q_{\text {Platenorm }}}=C q-\frac{1}{n} \sum_{i=1}^{n} C_{I C}
\end{array}
$$

Cq-values of the target miRNAs hsa-miR-96, hsa-miR$125 \mathrm{~b}$, hsa-miR-205, and hsa-miR-375 were further normalized to each selected reference gene and the relative concentration was calculated. The relative concentration of the miRNA in tumor tissue was further normalized to the relative expression of the same miRNA in normal tissue to determine the fold changes of differential expression in tumor tissue.

Normalization of the gene of interest (GOI) to reference gene:

$$
C_{G O I, n o r m}=C q_{G O I}-\frac{1}{n} \sum_{i=1}^{n} C q_{R G}
$$

Normalization of expression in tumor to expression in normal tissue:

$$
\text { Ratio } T / N=2^{-C q(\text { Tumor })+C q(\text { Normal })}
$$

Experiments were planned and realized according to the MIQE guidelines (Bustin et al., 2009).

\section{Statistical analysis}

Statistical analysis of qPCR data was performed with GraphPad Prism version 5.03 (GraphPad Software Inc., La Jolla, CA). The normality distribution of the data was tested by the Kolmogorov-Smirnov test. Parametric ( $t$-test according to Student with paired and unpaired values) and non-parametric tests (Wilcoxon signed rank test; MannWhitney U test; Friedman's test with Dunn's post test) were used as appropriate. Significant differences in variance were tested by Levene's F-test. $P$-values below 0.05 were regarded as significant.

\section{Acknowledgements}

This study was supported by the Foundation of Urologic Research, Berlin, Germany (AS) and the SONNENFELDStiftung Berlin, Germany (MJ; KJ).

\section{References}

Ambs S, Prueitt RL, Yi M, Hudson RS, Howe TM, Petrocca F, Wallace TA, Liu CG, Volinia S, Calin GA, Yfantis HG, Stephens RM, Croce CM. Genomic profiling of microRNA and messenger RNA reveals deregulated microRNA expression in prostate cancer. Cancer Res 2008;68:6162-70

Andersen CL, Jensen JL, Orntoft TF. Normalization of real-time quantitative reverse transcription-PCR data: a model-based variance estimation approach to identify genes suited for normalization, applied to bladder and colon cancer data sets. Cancer Res 2004;64:5245-50

Bartels CL, Tsongalis GJ. MicroRNAs: novel biomarkers for human cancer. Clin Chem 2009;55:623-31

Berezikov E, Guryev V, van de BJ, Wienholds E, Plasterk RH, Cuppen E. Phylogenetic shadowing and computational identification of human microRNA genes. Cell 2005;120: 21-4

Bolstad BM, Irizarry RA, Astrand M, Speed TP. A comparison of normalization methods for high density oligonucleotide array data based on variance and bias. Bioinformatics 2003;19:185-93

Bonci D, Coppola V, Musumeci M, Addario A, Giuffrida R, Memeo L, D'Urso L, Pagliuca A, Biffoni M, Labbaye C, Bartucci M, Muto G, Peschle C, De MR. The miR-15amiR-16-1 cluster controls prostate cancer by targeting multiple oncogenic activities. Nat Med 2008;14:1271-7

Bustin SA, Nolan T. Pitfalls of quantitative real-time reverse-transcription polymerase chain reaction. J Biomol Tech 2004;15:155-66

Bustin SA, Benes V, Garson JA, Hellemans J, Huggett J, Kubista M, Mueller R, Nolan T, Pfaffl MW, Shipley GL, Vandesompele J, Wittwer CT. The MIQE guidelines: minimum information for publication of quantitative real-time PCR experiments. Clin Chem 2009;55:611-22

Chang KH, Mestdagh P, Vandesompele J, Kerin MJ, Miller N. MicroRNA expression profiling to identify and validate reference genes for relative quantification in colorectal cancer. BMC Cancer 2010;10:173

Chen C, Ridzon DA, Broomer AJ, Zhou Z, Lee DH, Nguyen JT, Barbisin M, Xu NL, Mahuvakar VR, Andersen MR, Lao $K Q$, Livak KJ, Guegler KJ. Real-time quantification of microRNAs by stem-loop RT-PCR. Nucleic Acids Res 2005;33:e179

Davoren PA, McNeill RE, Lowery AJ, Kerin MJ, Miller N. Identification of suitable endogenous control genes for microRNA gene expression analysis in human breast cancer. BMC Mol Biol 2008;9:76

Dheda K, Huggett JF, Chang JS, Kim LU, Bustin SA, Johnson MA, Rook GA, Zumla A. The implications of using an inappropriate reference gene for real-time reverse transcription PCR data normalization. Anal Biochem 2005;344: 141-3

Epstein JI, Allsbrook WC Jr, Amin MB, Egevad LL. The 2005 International Society of Urological Pathology (ISUP) Consensus Conference on Gleason Grading of Prostatic 
Carcinoma. Am J Surg Pathol 2005;29:1228-42

Filipowicz W, Jaskiewicz L, Kolb FA, Pillai RS. Posttranscriptional gene silencing by siRNAs and miRNAs. Curr Opin Struct Biol 2005;15:331-41

Gandellini P, Folini M, Longoni N, Pennati M, Binda M, Colecchia M, Salvioni R, Supino R, Moretti R, Limonta P, Valdagni R, Daidone MG, Zaffaroni N. miR-205 Exerts tumor-suppressive functions in human prostate through down-regulation of protein kinase Cepsilon. Cancer Res 2009;69:2287-95

Garzon R, Calin GA, Croce CM. MicroRNAs in Cancer. Annu Rev Med 2009;60:167-79

Haller F, Kulle B, Schwager S, Gunawan B, von HA, Sultmann $H$, Fuzesi L. Equivalence test in quantitative reverse transcription polymerase chain reaction: confirmation of reference genes suitable for normalization. Anal Biochem 2004;335:1-9

Huang Q, Gumireddy K, Schrier M, le Sage C, Nagel R, Nair S, Egan DA, Li A, Huang G, Klein-Szanto AJ, Gimotty PA, Katsaros D, Coukos G, Zhang L, Pure E, Agami R. The microRNAs miR-373 and miR-520c promote tumour invasion and metastasis. Nat Cell Biol 2008;10:202-10

Jiang J, Lee EJ, Gusev Y, Schmittgen TD. Real-time expression profiling of microRNA precursors in human cancer cell lines. Nucleic Acids Res 2005;33:5394-403

Josson S, Sung SY, Lao K, Chung LW, Johnstone PA. Radiation modulation of microRNA in prostate cancer cell lines. Prostate 2008;68:1599-606

Jung M, Mollenkopf HJ, Grimm C, Wagner I, Albrecht M, Waller T, Pilarsky C, Johannsen M, Stephan C, Lehrach H, Nietfeld W, Rudel T, Jung K, Kristiansen G. MicroRNA profiling of clear cell renal cell cancer identifies a robust signature to define renal malignancy. J Cell Mol Med 2009;13:3918-28

Kubista M, Andrade JM, Bengtsson M, Forootan A, Jonak J, Lind K, Sindelka R, Sjoback R, Sjogreen B, Strombom L, Stahlberg A, Zoric N. The real-time polymerase chain reaction. Mol Aspects Med 2006;27:95-125

Lee YS, Dutta A. MicroRNAs in cancer. Annu Rev Pathol 2009;4:199-227

Leite KR, Sousa-Canavez JM, Reis ST, Tomiyama AH, Camara-Lopes LH, Sanudo A, Antunes AA, Srougi M. Change in expression of miR-let7c, miR-100, and miR-218 from high grade localized prostate cancer to metastasis. Urol Oncol 2009;in press:doi:10.1016/j.urolonc.2009.02.002

Mattie MD, Benz CC, Bowers J, Sensinger K, Wong L, Scott GK, Fedele V, Ginzinger D, Getts R, Haqq C. Optimized high-throughput microRNA expression profiling provides novel biomarker assessment of clinical prostate and breast cancer biopsies. Mol Cancer 2006;5:24

Mestdagh P, Van Vlierberghe P, De Weer A, Muth D, Westermann F, Speleman F, Vandesompele J. A novel and universal method for microRNA RT-qPCR data normalization. Genome Biol 2009;10:R64

Meyer SU, Pfaffl MW, Ulbrich SE. Normalization strategies for microRNA profiling experiments: a 'normal' way to a hidden layer of complexity? Biotechnol Lett 2010;32: 1777-88

Min H, Yoon S. Got target? Computational methods for microRNA target prediction and their extension. Exp Mol Med 2010;42:233-44

Mitchell PS, Parkin RK, Kroh EM, Fritz BR, Wyman SK, Pogosova-Agadjanyan EL, Peterson A, Noteboom J, O'Briant KC, Allen A, Lin DW, Urban N, Drescher CW, Knudsen BS, Stirewalt DL, Gentleman R, Vessella RL, Nelson PS, Martin DB, Tewari M. Circulating microRNAs as stable blood-based markers for cancer detection. Proc Natl Acad Sci USA 2008;105:10513-8

Nolan T, Hands RE, Bustin SA. Quantification of mRNA using real-time RT-PCR. Nat Protoc 2006;1:1559-82

Noonan EJ, Place RF, Pookot D, Basak S, Whitson JM, Hirata H, Giardina C, Dahiya R. miR-449a targets HDAC-1 and induces growth arrest in prostate cancer. Oncogene 2009;28:1714-24

Ohl F, Jung M, Xu C, Stephan C, Rabien A, Burkhardt M, Nitsche A, Kristiansen G, Loening SA, Radonic A, Jung K. Gene expression studies in prostate cancer tissue: which reference gene should be selected for normalization? J Mol Med 2005;83:1014-24

Ozen M, Creighton CJ, Ozdemir M, Ittmann M. Widespread deregulation of microRNA expression in human prostate cancer. Oncogene 2008;27:1788-93

Peltier HJ, Latham GJ. Normalization of microRNA expression levels in quantitative RT-PCR assays: identification of suitable reference RNA targets in normal and cancerous human solid tissues. RNA 2008;14:844-52

Place RF, Li LC, Pookot D, Noonan EJ, Dahiya R. MicroRNA373 induces expression of genes with complementary promoter sequences. Proc Natl Acad Sci USA 2008;105: 1608-13

Porkka KP, Pfeiffer MJ, Waltering KK, Vessella RL, Tammela TL, Visakorpi T. MicroRNA expression profiling in prostate cancer. Cancer Res 2007;67:6130-5

Pradervand S, Weber J, Thomas J, Bueno M, Wirapati P, Lefort K, Dotto GP, Harshman K. Impact of normalization on miRNA microarray expression profiling. RNA 2009;15:493501

Prueitt RL, Yi M, Hudson RS, Wallace TA, Howe TM, Yfantis HG, Lee DH, Stephens RM, Liu CG, Calin GA, Croce CM, Ambs S. Expression of microRNAs and protein-coding genes associated with perineural invasion in prostate cancer. Prostate 2008;68:1152-64

Rokhlin OW, Scheinker VS, Taghiyev AF, Bumcrot D, Glover RA, Cohen MB. MicroRNA-34 mediates AR-dependent p53-induced apoptosis in prostate cancer. Cancer Biol Ther 2008;7:1288-96

Rondinelli RH, Epner DE, Tricoli JV. Increased glyceraldehyde-3-phosphate dehydrogenase gene expression in late pathological stage human prostate cancer. Prostate Cancer Prostatic Dis 1997;1:66-72

Schaefer A, Jung M, Mollenkopf H-J, Wagner I, Stephan C, Jentzmik F, Miller K, Lein M, Kristiansen $G$, Jung $K$. 
Diagnostic and prognostic implications of microRNA profiling in prostate carcinoma. Int J Cancer 2010a;126:1166-76

Schaefer A, Stephan C, Busch J, Yousef GM, Jung K. Diagnostic, prognostic, and therapeutic implications of miRNAs in urologic tumors. Nat Rev Urol 2010b;7:286-97

Siva AC, Nelson LJ, Fleischer CL, Majlessi M, Becker MM, Vessella RL, Reynolds MA. Molecular assays for the detection of microRNAs in prostate cancer. Mol Cancer 2009;8:17

Sobin LH, Wittekind C. TNM Classification of Malignant Tumours, 6th Ed, 2002, Wiley-Liss, New York, NY.

Spahn M, Kneitz S, Scholz CJ, Nico S, Rudiger T, Strobel P,
Riedmiller $\mathrm{H}$, Kneitz B. Expression of microRNA-221 is progressively reduced in aggressive prostate cancer and metastasis and predicts clinical recurrence. Int J Cancer 2010;127:394-403

Tong AW, Fulgham P, Jay C, Chen P, Khalil I, Liu S, Senzer $\mathrm{N}$, Eklund AC, Han J, Nemunaitis J. MicroRNA profile analysis of human prostate cancers. Cancer Gene Ther 2009;16:206-16

Vandesompele J, De Preter K, Pattyn F, Poppe B, Van Roy $N$, De Paepe A, Speleman F. Accurate normalization of real-time quantitative RT-PCR data by geometric averaging of multiple internal control genes. Genome Biol 2002;3: RESEARCH0034 